\title{
On the Ulam stability of a class of Banach space valued linear differential equations of second order
}

\author{
Yonghong Shen ${ }^{1,2^{*}}$, Wei Chen ${ }^{3}$ and Yaoyao Lan ${ }^{4}$
}

\section{"Correspondence:}

shenyonghong2008@hotmail.com

'School of Mathematics and

Statistics, Tianshui Normal

University, Tianshui, 741001, P.R. China

${ }^{2}$ School of Mathematics and Statistics, Beijing Institute of Technology, Beijing, 100081, P.R. China

Full list of author information is available at the end of the article

\begin{abstract}
Let $E$ be a complex Banach space. We prove the Ulam stability of a class of Banach space valued second order linear differential equations $p(x) y^{\prime \prime}(x)+q(x) y^{\prime}(x)+\lambda y(x)=0$, where $p \in C^{1}\left[I, \mathbb{R}^{+}\right], q \in C[I, \mathbb{R}]$ with $p^{\prime}(x)=2 q(x)$ for each $x \in l$; / denotes an open interval in $\mathbb{R}, \lambda$ is a fixed positive real number. Moreover, we also provide some applications of our results.
\end{abstract}

Keywords: Ulam stability; linear differential equation; Chebyshev differential equation; Banach space

\section{Introduction}

At present, the Ulam stability (Hyers-Ulam stability or Hyers-Ulam-Rassias stability) is one of the most active research topics in the theory of functional equations. The study of such stability problems for functional equations originated from a question of Ulam [1] concerning the stability of group homomorphisms. Hyers [2] gave a first affirmative partial answer to this question for Banach spaces. Afterwards, Rassias [3] generalized the result of Hyers [2] for linear mappings in which the Cauchy difference is allowed to be unbounded, and this work has great influence on the development of this type of stability theory of functional equations. Since then, the stability problems for various functional equations have been extensively studied. For more details, the reader is referred to $[4,5]$.

In 1993, Obloza [6] initiated the investigation on the Hyers-Ulam stability of differential equations. Later, Alsina and Ger [7] proved that the Hyers-Ulam stability of the differential equation $y^{\prime}=y$ holds. Specifically, for a given $\epsilon \geq 0$, if $f$ is a differentiable function on an open subinterval $I$ into $\mathbb{R}$ with $\left|f^{\prime}(x)-f(x)\right| \leq \epsilon$ for all $x \in I$, then there exists a differentiable function $g: I \rightarrow \mathbb{R}$ such that $g^{\prime}(t)=g(t)$ and satisfying $|f(x)-g(x)| \leq 3 \epsilon$ for all $x \in I$. Soon after, the above result was generalized by Miura and Takahasi et al. [8-10]. Up to the present, the Hyers-Ulam stability or Hyers-Ulam-Rassias stability of the first order and higher order linear differential equations have been widely and extensively investigated by many authors [11-20].

Several years ago, Jung together with Kim and Rassias [21, 22] discussed the general solution of the complex-valued Chebyshev differential equation $\left(1-x^{2}\right) y^{\prime \prime}(x)-x y^{\prime}(x)+n^{2} y(x)=$ 0 and studied its Hyers-Ulam stability. Hereafter, Miura et al. [23] further improved the 
stability result of Chebyshev differential equation. Inspired by the work of Miura et al., we will start the following work.

Let $E$ be a complex Banach space with a norm $\|\cdot\|$. Unless otherwise stated, let $I=$ $(a, b), a, b \in \mathbb{R} \cup\{ \pm \infty\}$, denote the open interval of $\mathbb{R}$. In addition, we denote by $C[I, E]$ and $C^{1}[I, E]\left(C^{2}[I, E]\right)$ the set of all $E$-valued continuous functions on $I$ and the set of all strongly (two times) differentiable functions which have a continuous (second) derivative on $I$, respectively. In a more general framework, we shall investigate the Ulam stability of the following second order linear differential equation:

$$
p(x) y^{\prime \prime}(x)+q(x) y^{\prime}(x)+\lambda y(x)=0
$$

where $p \in C^{1}\left[I, \mathbb{R}^{+}\right]$(here $\mathbb{R}^{+}$denotes the set of all positive real numbers), $q \in C[I, \mathbb{R}]$ with $p^{\prime}(x)=2 q(x)$ for each $x \in I, \lambda$ is a fixed positive real number, $y \in C^{2}[I, E]$.

\section{Main results}

For a $n$-order $E$-valued differential equation

$$
F\left(x, y, y^{\prime}, \ldots, y^{(n)}\right)=0, \quad x \in I
$$

we say that it has the Hyers-Ulam stability or it is stable in the sense of Hyers-Ulam sense if for a given $\epsilon>0$ and a $n$ times strongly differentiable function $f: I \rightarrow E$ satisfying $\left\|F\left(x, f, f^{\prime}, \ldots, f^{(n)}\right)\right\| \leq \epsilon$ for all $x \in I$, then there exists an exact solution $h$ of this equation such that $\|f(x)-h(x)\| \leq K(\epsilon)$ for all $x \in I$, where $K(\epsilon)$ depends only on $\epsilon$, and $\lim _{\epsilon \rightarrow 0} K(\epsilon)=0$. More generally, if $\epsilon$ and $K(\epsilon)$ are replaced by two control functions $\varphi, \Phi: I \rightarrow[0,+\infty)$, respectively, we say that the above differential equation has the HyersUlam-Rassias stability or it is stable in the sense of Hyers-Ulam-Rassias.

The following theorem is the main result of this paper.

Theorem 2.1 Let $\varphi: I \rightarrow[0, \infty)$ be a function such that $\varphi(x)$ is integrable on the interval $[r, s]$ for each $r, s \in I$ with $r \leq s$. Suppose that $f \in C^{2}[I, E]$ satisfies the differential inequality

$$
\left\|p(x) f^{\prime \prime}(x)+q(x) f^{\prime}(x)+\lambda f(x)\right\| \leq \varphi(x)
$$

for all $x \in I$. Moreover, assume that the equation

$$
\omega^{\prime}(t)=(p(\omega(t)))^{\frac{1}{2}}
$$

has a solution on $J=(c, d)$, where $t \in J$ corresponds to $\omega(t) \in I$. Then there exists $h \in C^{2}[I, E]$ such that $h$ satisfies Eq. (1) and

$$
\|f(x)-h(x)\| \leq\left|\int_{a_{0}}^{\omega^{-1}(x)} \int_{a_{0}}^{\omega^{-1}(\rho)} \frac{\varphi(\sigma)}{\omega^{\prime}\left(\omega^{-1}(\sigma)\right)} \cdot \frac{1}{\omega^{\prime}\left(\omega^{-1}(\rho)\right)} d \sigma d \rho\right|
$$

for all $x \in I$, where $a_{0} \in I$ is an arbitrary fixed point.

Proof Firstly, we take the solution $\omega(t)$ of Eq. (3) and make an appropriate substitution for the variable $x$. Obviously, the condition $\omega^{\prime}(t)>0, t \in J$, implies that the inverse function $t=\omega^{-1}(x)$ exists on $I$. 
Now, we define the map $\eta: J \rightarrow E$ by $\eta(t):=f(\omega(t))$ for each $t \in J$. Obviously, $\eta \in C^{2}[J, E]$. Then we can obtain

$$
\eta^{\prime}(t)=\omega^{\prime}(t) f^{\prime}(\omega(t)), \quad \eta^{\prime \prime}(t)=\omega^{\prime \prime}(t) f^{\prime}(\omega(t))+\omega^{\prime}(t)^{2} f^{\prime \prime}(\omega(t)) .
$$

Moreover, since $p^{\prime}(x)=2 q(x)$, we get $p^{\prime}(\omega(t))=2 q(\omega(t))$ for each $t \in J$. Thus, it follows from Eq. (3) that

$$
2 \omega^{\prime}(t) \omega^{\prime \prime}(t)=p^{\prime}(\omega(t)) \omega^{\prime}(t)=2 \omega^{\prime}(t) q(\omega(t)),
$$

which implies that $\omega^{\prime \prime}(t)=q(\omega(t))$ for each $t \in J$.

Therefore, we have

$$
\begin{aligned}
\eta^{\prime \prime}(t)+\lambda \eta(t) & =\omega^{\prime}(t)^{2} f^{\prime \prime}(\omega(t))+\omega^{\prime \prime}(t) f^{\prime}(\omega(t))+\lambda f(\omega(t)) \\
& =p(\omega(t)) f^{\prime \prime}(\omega(t))+q(\omega(t)) f^{\prime}(\omega(t))+\lambda f(\omega(t))
\end{aligned}
$$

for all $t \in J$. Note that $\omega(t) \in I$ for each $t \in J$. Based on the inequality (2) and the preceding equality, we can obtain

$$
\left\|\eta^{\prime \prime}(t)+\lambda \eta(t)\right\| \leq \varphi(\omega(t))
$$

for all $t \in J$.

We set $\alpha(t)=\eta^{\prime \prime}(t)+\lambda \eta(t), \beta(t)=\eta^{\prime}(t)+i \sqrt{\lambda} \eta(t)$. Then we have

$$
\begin{aligned}
\beta^{\prime}(t) & =\eta^{\prime \prime}(t)+i \sqrt{\lambda} \eta^{\prime}(t) \\
& =(\alpha(t)-\lambda \eta(t))+i \sqrt{\lambda} \eta^{\prime}(t) \\
& =i \sqrt{\lambda}\left(\eta^{\prime}(t)+i \sqrt{\lambda} \eta(t)\right)+\alpha(t) \\
& =i \sqrt{\lambda} \beta(t)+\alpha(t) .
\end{aligned}
$$

It follows that $\beta^{\prime}(t)-i \sqrt{\lambda} \beta(t)=\alpha(t)$. Multiplying both sides of the previous equality by $e^{-i \sqrt{\lambda} t}, t \in J$, we obtain

$$
\left(e^{-i \sqrt{\lambda} t} \beta(t)\right)^{\prime}=e^{-i \sqrt{\lambda} t}\left(\beta^{\prime}(t)-i \sqrt{\lambda} \beta(t)\right)=e^{-i \sqrt{\lambda} t} \alpha(t) .
$$

By integrating both sides of the above equality from $c_{0}$ (here $c_{0}$ is an arbitrary fixed point in $J$ ) to $s$ with respect to $\tau, c_{0}, s \in J$, it follows that

$$
\begin{aligned}
\int_{c_{0}}^{s} e^{-i \sqrt{\lambda} \tau} \alpha(\tau) d \tau & =\int_{c_{0}}^{s}\left(e^{-i \sqrt{\lambda} \tau} \beta(\tau)\right)^{\prime} d \tau \\
& =e^{-i \sqrt{\lambda} s} \beta(s)-e^{-i \sqrt{\lambda} c_{0}} \beta\left(c_{0}\right) .
\end{aligned}
$$

Furthermore, we can infer that

$$
\beta(s)=e^{i \sqrt{\lambda} s}\left(e^{-i \sqrt{\lambda} c_{0}} \beta\left(c_{0}\right)+\int_{c_{0}}^{s} e^{-i \sqrt{\lambda} \tau} \alpha(\tau) d \tau\right)
$$

for all $s \in J$. 
In view of $\beta(s)=\eta^{\prime}(s)+i \sqrt{\lambda} \eta(s)$, for each $s \in J$, we have

$$
e^{i \sqrt{\lambda s}} \beta(s)=e^{i \sqrt{\lambda s}}\left(\eta^{\prime}(s)+i \sqrt{\lambda} \eta(s)\right)=\left(e^{i \sqrt{\lambda} s} \eta(s)\right)^{\prime} .
$$

Analogously, by integrating both sides of the last equality from $c_{0}$ to $t$ with respect to $s$, we conclude that

$$
\begin{aligned}
e^{i \sqrt{\lambda} t} \eta(t)-e^{i \sqrt{\lambda} c_{0}} \eta\left(c_{0}\right) & =\int_{c_{0}}^{t}\left(e^{i \sqrt{\lambda} s} \eta(s)\right)^{\prime} d s \\
& =\int_{c_{0}}^{t} e^{i \sqrt{\lambda} s} \beta(s) d s \\
& =\int_{c_{0}}^{t} e^{2 i \sqrt{\lambda} s}\left(e^{-i \sqrt{\lambda} c_{0}} \beta\left(c_{0}\right)+\int_{c_{0}}^{s} e^{-i \sqrt{\lambda} \tau} \alpha(\tau) d \tau\right) d s \\
& =\frac{e^{i \sqrt{\lambda}\left(2 t-c_{0}\right)}-e^{i \sqrt{\lambda} c_{0}}}{2 \sqrt{\lambda} i} \beta\left(c_{0}\right)+\int_{c_{0}}^{t} \int_{c_{0}}^{s} e^{-i \sqrt{\lambda}(\tau-2 s)} \alpha(\tau) d \tau d s .
\end{aligned}
$$

Define the map $g: J \rightarrow E$ by

$$
g(t):=e^{-i \sqrt{\lambda}\left(t-c_{0}\right)} \eta\left(c_{0}\right)+\frac{e^{i \sqrt{\lambda}\left(t-c_{0}\right)}-e^{-i \sqrt{\lambda}\left(t-c_{0}\right)}}{2 \sqrt{\lambda} i} \beta\left(c_{0}\right), \quad t \in J .
$$

Clearly, $g \in C^{2}[J, E]$. Therefore, we can infer that

$$
g^{\prime}(t)=-i \sqrt{\lambda} e^{-i \sqrt{\lambda}\left(t-c_{0}\right)} \eta\left(c_{0}\right)+\frac{i \sqrt{\lambda} e^{i \sqrt{\lambda}\left(t-c_{0}\right)}+i \sqrt{\lambda} e^{-i \sqrt{\lambda}\left(t-c_{0}\right)}}{2 \sqrt{\lambda} i} \beta\left(c_{0}\right),
$$

and thus

$$
g^{\prime \prime}(t)=-\lambda e^{-i \sqrt{\lambda}\left(t-c_{0}\right)} \eta\left(c_{0}\right)+\frac{-\lambda e^{i \sqrt{\lambda}\left(t-c_{0}\right)}+\lambda e^{-i \sqrt{\lambda}\left(t-c_{0}\right)}}{2 \sqrt{\lambda} i} \beta\left(c_{0}\right) .
$$

From the equalities (7) and (8), it is easy to see that $g^{\prime \prime}(t)=-\lambda g(t)$ for each $t \in J$. Furthermore, we can infer from Eq. (6) that

$$
\begin{aligned}
e^{i \sqrt{\lambda} t} \eta(t) & =e^{i \sqrt{\lambda} c_{0}} \eta\left(c_{0}\right)+\frac{e^{i \sqrt{\lambda}\left(2 t-c_{0}\right)}-e^{i \sqrt{\lambda} c_{0}}}{2 \sqrt{\lambda} i} \beta\left(c_{0}\right)+\int_{c_{0}}^{t} \int_{c_{0}}^{s} e^{-i \sqrt{\lambda}(\tau-2 s)} \alpha(\tau) d \tau d s \\
& =e^{i \sqrt{\lambda} t} g(t)+\int_{c_{0}}^{t} \int_{c_{0}}^{s} e^{-i \sqrt{\lambda}(\tau-2 s)} \alpha(\tau) d \tau d s
\end{aligned}
$$

for each $t \in J$. Then, by Eq. (5), we can obtain

$$
\begin{aligned}
\|\eta(t)-g(t)\| & =\mid e^{-i \sqrt{\lambda} t} \int_{c_{0}}^{t} \int_{c_{0}}^{s} e^{-i \sqrt{\lambda}(\tau-2 s)} \alpha(\tau) d \tau d s \| \\
& \leq\left|\int_{c_{0}}^{t} \int_{c_{0}}^{s}\|\alpha(\tau)\| d \tau d s\right| \\
& \leq\left|\int_{c_{0}}^{t} \int_{c_{0}}^{s} \varphi(\omega(\tau)) d \tau d s\right|
\end{aligned}
$$


Finally, we define the map $h: I \rightarrow E$ by

$$
h(x)=g\left(\omega^{-1}(x)\right), \quad x \in I .
$$

Therefore, we get

$$
h^{\prime}(x)=g^{\prime}\left(\omega^{-1}(x)\right)\left(\omega^{-1}(x)\right)^{\prime} .
$$

Furthermore, we can obtain

$$
h^{\prime \prime}(x)=\left(\omega^{-1}(x)\right)^{\prime \prime} g^{\prime}\left(\omega^{-1}(x)\right)+\left[\left(\omega^{-1}(x)\right)^{\prime}\right]^{2} g^{\prime \prime}\left(\omega^{-1}(x)\right) .
$$

According to the derivative of the inverse function, it follows that

$$
\left(\omega^{-1}(x)\right)^{\prime}=\frac{1}{\omega^{\prime}(t)}, \quad\left(\omega^{-1}(x)\right)^{\prime \prime}=\frac{-\omega^{\prime \prime}(t)}{\left[\omega^{\prime}(t)\right]^{2}} .
$$

Moreover, in view of $\omega^{\prime}(t)=[p(\omega(t))]^{\frac{1}{2}}$ together with $\omega(t)$ being a solution of Eq. (3), we can infer from Eq. (12) that

$$
\left(\omega^{-1}(x)\right)^{\prime}=(p(x))^{-\frac{1}{2}}, \quad\left(\omega^{-1}(x)\right)^{\prime \prime}=-q(x)(p(x))^{-\frac{3}{2}} .
$$

Then it follows from Eqs. (8), (10), (11), and (13) that

$$
\begin{aligned}
h^{\prime \prime}(x) & =-q(x) p(x)^{-\frac{3}{2}} g^{\prime}\left(\omega^{-1}(x)\right)+p(x)^{-1} g^{\prime \prime}\left(\omega^{-1}(x)\right) \\
& =p(x)^{-1}\left(-q(x) p(x)^{-\frac{1}{2}} g^{\prime}\left(\omega^{-1}(x)\right)+g^{\prime \prime}\left(\omega^{-1}(x)\right)\right) \\
& =p(x)^{-1}\left(-q(x) h^{\prime}(x)-\lambda g\left(\omega^{-1}(x)\right)\right) \\
& =p(x)^{-1}\left(-q(x) h^{\prime}(x)-\lambda h(x)\right) .
\end{aligned}
$$

Hence, we get

$$
p(x) h^{\prime \prime}(x)+q(x) h^{\prime}(x)+\lambda h(x)=0
$$

for all $x \in I$. This means that $h \in C^{2}(I, E)$ is a solution of Eq. (1).

Recall that $\eta(t)=f(\omega(t))$ for each $t \in J$, namely, $f(x)=\eta\left(\omega^{-1}(x)\right)$ for each $x \in I$. By Eq. (9), we can obtain

$$
\begin{aligned}
\|f(x)-h(x)\| & =\left\|\eta\left(\omega^{-1}(x)\right)-g\left(\omega^{-1}(x)\right)\right\| \\
& \leq\left|\int_{a_{0}}^{\omega^{-1}(x)} \int_{a_{0}}^{\omega^{-1}(\rho)} \frac{\varphi(\sigma)}{\omega^{\prime}\left(\omega^{-1}(\sigma)\right)} \cdot \frac{1}{\omega^{\prime}\left(\omega^{-1}(\rho)\right)} d \sigma d \rho\right|,
\end{aligned}
$$

where $a_{0}=\omega^{-1}\left(c_{0}\right) \in I$.

Remark 1 In Theorem 2.1, the arbitrariness of $a_{0}$ does not mean that the right-hand side of the inequality (4) may be arbitrarily changed with respect to an appropriate function $h$, 
because one can see from Eq. (7) that the desired function $h$ depends on the choice of $a_{0}$. Furthermore, we can conclude from Eq. (4) that for an arbitrary fixed $a_{0} \in I$, there exists an appropriate solution $h$ of Eq. (1) such that $h$ can be used to approximate the function $f$ that satisfies the inequality (2), and the error can be estimated by the control function of the right-hand side of Eq. (4).

The following result associated with the Hyers-Ulam stability of Eq. (1) is a direct consequence of Theorem 2.1.

Corollary 2.2 Let $I=(a, b)$ be a finite interval, i.e., $-\infty<a<b<+\infty$ and let $\epsilon>0$ be $a$ given number. Suppose that $f \in C^{2}[I, E]$ satisfies the differential inequality

$$
\left\|p(x) f^{\prime \prime}(x)+q(x) f^{\prime}(x)+\lambda f(x)\right\| \leq \epsilon
$$

for all $x \in I$. Moreover, assume that Eq. (3) has a solution $\omega(t)$ on $J=(c, d)$, where $t \in J$ corresponds to $\omega(t) \in I$. Then there exists $h \in C^{2}[I, E]$ such that $h$ satisfies Eq. (1) and

$$
\|f(x)-h(x)\| \leq \frac{(d-c)^{2}}{2} \epsilon
$$

for all $x \in I$.

Proof According to Theorem 2.1, it suffices to verify that the inequality (15) holds for all $x \in I$. By Eqs. (5) and (9), we have

$$
\begin{aligned}
\|f(x)-h(x)\| & =\|\eta(t)-g(t)\| \\
& \leq\left|\int_{c_{0}}^{t} \int_{c_{0}}^{s}\|\alpha(\tau)\| d \tau d s\right| \\
& \leq \frac{\left(t-c_{0}\right)^{2}}{2} \epsilon \\
& <\frac{(d-c)^{2}}{2} \epsilon
\end{aligned}
$$

for all $x \in I$.

Remark 2 In Corollary 2.2, since $c_{0} \in(c, d)$ is an arbitrary fixed point, the right-hand side of the equality (15) can be further improved. In fact, since $t \in(c, d)$ is arbitrary, if we take the midpoint of the interval $(c, d)$, i.e., putting $c_{0}=\frac{c+d}{2}$, then we can obtain a better upper bound of the equality (15). Furthermore, the inequality (15) can be improved as

$$
\|f(x)-h(x)\| \leq \frac{(d-c)^{2}}{8} \epsilon
$$

\section{Applications}

In this section, some practical examples are given to illustrate the main results proposed in the previous section. 
Example 1 Let $E$ be a complex Banach space. Then the differential equation

$$
\left(m^{2}-x^{2}\right) y^{\prime \prime}(x)-x y^{\prime}(x)+\lambda y(x)=0
$$

has the Hyers-Ulam stability when $x \in I=(-m, m)$, where $m, \lambda \in \mathbb{R}^{+}$. More precisely, for each $\epsilon>0$, if $f \in C^{2}[I, E]$ satisfies the following differential inequality:

$$
\left\|\left(m^{2}-x^{2}\right) f^{\prime \prime}(x)-x f^{\prime}(x)+\lambda f(x)\right\| \leq \epsilon
$$

for all $x \in I$, then there exists $h \in C^{2}[I, E]$ such that $h$ satisfies Eq. (17) and

$$
\|f(x)-h(x)\| \leq \frac{\pi^{2}}{8} \epsilon
$$

for all $x \in I$.

According to Corollary 2.2, we take $p(x)=m^{2}-x^{2}, q(x)=-x$. Obviously, we have $p^{\prime}(x)=$ $2 q(x)$ for each $x \in I$. Consider the equation $\omega^{\prime}(t)=\sqrt{m^{2}-\omega(t)^{2}}$. It is easy to know that $\omega(t)=m \sin t$ is a solution on $J=\left(-\frac{\pi}{2}, \frac{\pi}{2}\right)$. Clearly, it is easy to check that $\omega(t)=m \sin t$ satisfies the condition of Corollary 2.2, and hence there exists $h \in C^{2}[I, E]$ such that $h$ satisfies Eq. (17) and the inequality (18).

Remark 3 In Example 1, if $m=1, \lambda=n^{2}, n \in \mathbb{N}$ (here $\mathbb{N}$ denotes the set of all natural numbers), Eq. (17) will degenerate into the Chebyshev differential equation. Therefore, the main results obtained in [23] will be included in Example 1 as a special case.

Example 2 Let $E$ be a complex Banach space. Then the differential equation

$$
x^{2} y^{\prime \prime}(x)+x y^{\prime}(x)+\lambda y(x)=0
$$

has the Hyers-Ulam-Rassias stability when $x \in I=(0,+\infty)$, where $\lambda \in \mathbb{R}^{+}$. Specifically, for each $\epsilon>0$, if $f \in C^{2}[I, E]$ satisfies the following differential inequality:

$$
\left\|x^{2} f^{\prime \prime}(x)+x f^{\prime}(x)+\lambda f(x)\right\| \leq \epsilon
$$

for all $x \in I$, then there exists $h \in C^{2}[I, E]$ such that $h$ satisfies Eq. (19) and

$$
\|f(x)-h(x)\| \leq \frac{\left(\ln x-a_{0}\right)^{2}}{2} \epsilon
$$

for all $x \in I$, where $a_{0}$ is an arbitrary fixed point in $I$.

Let $p(x)=x^{2}, q(x)=x$. Clearly, $p^{\prime}(x)=2 q(x)$ for each $x \in I$. Consider the differential equation $\omega^{\prime}(t)=\omega(t)$. It can easily be verified that $\omega(t)=A e^{t}(A>0)$ is a solution on $J=(-\infty,+\infty)$. For simplicity, we take $A=1$. Therefore, $x=e^{t}$ is an appropriate substitution for the variable $x$. According to Theorem 2.1, there exists $h \in C^{2}[I, E]$ such that $h$ 
satisfies Eq. (19). Moreover, it follows from Eq. (9) that

$$
\begin{aligned}
\|f(x)-h(x)\| & \leq\left|\int_{c_{0}}^{t} \int_{c_{0}}^{s} \epsilon d \tau d s\right| \\
& =\frac{\left(t-c_{0}\right)^{2}}{2} \epsilon=\frac{\left(\ln x-a_{0}\right)^{2}}{2} \epsilon \quad\left(x=e^{t}\right)
\end{aligned}
$$

for all $x \in I$. This implies that the inequality (20) holds.

Next, we shall further consider a more general example as a complement of Example 2.

Example 3 Let $E$ be a complex Banach space. For each $\epsilon>0$, assume that a function $f \in C^{2}[I, E]$ satisfies the following differential inequality:

$$
\left\|\left(m^{2}+x^{2}\right) f^{\prime \prime}(x)+x f^{\prime}(x)+\lambda f(x)\right\| \leq \epsilon
$$

for all $x \in I=(-\infty,+\infty)$, where $m, \lambda \in \mathbb{R}^{+}$. Then there exists $h \in C^{2}[I, E]$ such that

$$
\left(m^{2}+x^{2}\right) h^{\prime \prime}(x)+x h^{\prime}(x)+\lambda h(x)=0
$$

and

$$
\|f(x)-h(x)\| \leq \frac{\left(\ln \left(x+\sqrt{m^{2}+x^{2}}\right)-a_{0}\right)^{2}}{2} \epsilon
$$

for all $x \in I$, where $a_{0}$ is an arbitrary fixed point in $I$.

Let $p(x)=m^{2}+x^{2}, q(x)=x$. Consider the differential equation $\omega^{\prime}(t)=\sqrt{m^{2}+\omega(t)^{2}}$. Based on the theory of ordinary differential equations, we see that $x=m \sinh (t)$ is a solution on $J=(-\infty,+\infty)$, where $t \in J$ corresponds to $\omega(t) \in I$. Therefore, one can see that $x=$ $m \sinh (t)$ is an appropriate substitution for the variable $x$. By Theorem 2.1 , there exists $h \in C^{2}[I, E]$ such that the equality (21) holds and

$$
\begin{aligned}
\|f(x)-h(x)\| & \leq \frac{\left(t-c_{0}\right)^{2}}{2} \epsilon \\
& =\frac{\left(\ln \left(x+\sqrt{m^{2}+x^{2}}\right)-a_{0}\right)^{2}}{2} \epsilon \quad\left(\sinh ^{-1}(x)=\ln \left(x+\sqrt{1+x^{2}}\right)\right)
\end{aligned}
$$

for all $x \in I$, where $a_{0}=\ln m+\sinh \left(c_{0}\right)$.

The authors declare that they have no competing interests.

Authors' contributions

All authors contributed equally and significantly in writing this article. All authors read and approved the final manuscript.

\section{Author details}

${ }^{1}$ School of Mathematics and Statistics, Tianshui Normal University, Tianshui, 741001, P.R. China. ${ }^{2}$ School of Mathematics and Statistics, Beijing Institute of Technology, Beijing, 100081, P.R. China. ${ }^{3}$ School of Information, Capital University of Economics and Business, Beijing, 100070, P.R. China. ${ }^{4}$ Department of Mathematics and Finance, Chongqing University of Arts and Sciences, Yongchuan, 402160, P.R. China. 


\section{Acknowledgements}

This work was supported by 'Qing Lan' Talent Engineering Funds by Tianshui Normal University. The second author acknowledges the support of the Humanity and Social Science Youth Foundation of Ministry of Education of China (no. 13YJC630012), and the Specially Commissioned Project of the Capital University of Economics and Business. The third author acknowledges the support of the National Natural Science Foundation of China (no. 11226268).

Received: 18 September 2014 Accepted: 12 November 2014 Published: 25 Nov 2014

\section{References}

1. Ulam, SM: Problems in Modern Mathematics. Wiley, New York (1960)

2. Hyers, DH: On the stability of the linear functional equation. Proc. Natl. Acad. Sci. USA 27, 222-224 (1941)

3. Rassias, TM: On the stability of the linear mapping in Banach spaces. Proc. Am. Math. Soc. 72, 297-300 (1978)

4. Jung, SM: Hyers-Ulam-Rassias Stability of Functional Equations in Nonlinear Analysis. Springer, Berlin (2011)

5. Rassias, TM: On the stability of functional equations and a problem of Ulam. Acta Appl. Math. 62, 23-130 (2000)

6. Obloza, M: Hyers stability of the linear differential equation. Rocznik Nauk.-Dydakt. Prace Mat. 13, 259-270 (1993)

7. Alsina, C, Ger, R: On some inequalities and stability results related to the exponential function. J. Inequal. Appl. 2, 373-380 (1998)

8. Miura, T, Takahasi, SE, Choda, $\mathrm{H}$ : On the Hyers-Ulam stability of real continuous function valued differentiable map. Tokyo J. Math. 24, 467-476 (2001)

9. Miura, T: On the Hyers-Ulam stability of a differentiable map. Sci. Math. Jpn. 55, 17-24 (2002)

10. Takahasi, SE, Miura, T, Miyajima, S: On the Hyers-Ulam stability of the Banach space-valued differential equation $y^{\prime}=\lambda y$. Bull. Korean Math. Soc. 39, 309-315 (2002)

11. Abdollahpour, MR, Najati, A: Stability of linear differential equations of third order. Appl. Math. Lett. 24, 1827-1830 (2011)

12. Cimpean, DS, Popa, D: On the stability of the linear differential equation of higher order with constant coefficients. Appl. Math. Comput. 217, 4141-4146 (2010)

13. Jung, SM: Hyers-Ulam stability of linear differential equations of first order. Appl. Math. Lett. 17, 1135-1140 (2004)

14. Jung, SM: Hyers-Ulam stability of linear differential equations of first order (II). Appl. Math. Lett. 19, 854-858 (2006)

15. Jung, SM: Hyers-Ulam stability of linear differential equations of first order (III). J. Math. Anal. Appl. 311, 139-146 (2005)

16. Li, Y, Shen, Y: Hyers-Ulam stability of linear differential equations of second order. Appl. Math. Lett. 23, 306-309 (2010)

17. Miura, T, Miyajima, S, Takahasi, SE: A characterization of Hyers-Ulam stability of first order linear differential operators. J. Math. Anal. Appl. 286, 136-146 (2003)

18. Popa, D, Raşa, I: On the Hyers-Ulam stability of the linear differential equation. J. Math. Anal. Appl. 381, 530-537 (2011)

19. Popa, D, Raşa, I: Hyers-Ulam stability of the linear differential operator with non-constant coefficients. Appl. Math. Comput. 219, 1562-1568 (2012)

20. Takahasi, SE, Takagi, H, Miura, T, Miyajima, S: The Hyers-Ulam stability constants of first order linear differential operators. J. Math. Anal. Appl. 296, 403-409 (2004)

21. Jung, SM, Kim, B: Chebyshev's differential equation and its Hyers-Ulam stability. Differ. Equ. Appl. 1, 199-207 (2009)

22. Jung, SM, Rassias, TM: Approximation of analytic functions by Chebyshev functions. Abstr. Appl. Anal. 2011, Article ID 432961 (2011)

23. Miura, T, Yakahasi, SE, Hayata, T, Tanahashi, K: Stability of the Banach space valued Chebyshev differential equation. Appl. Math. Lett. 25, 1976-1979 (2012)

10.1186/1687-1847-2014-294

Cite this article as: Shen et al:: On the Ulam stability of a class of Banach space valued linear differential equations of second order. Advances in Difference Equations 2014, 2014:294

\section{Submit your manuscript to a SpringerOpen ${ }^{\odot}$ journal and benefit from:}

- Convenient online submission

- Rigorous peer review

- Immediate publication on acceptance

- Open access: articles freely available online

- High visibility within the field

- Retaining the copyright to your article 